\title{
Autoimmune polyendocrinopathy type 3
}

INSERM

\section{Source}

INSERM. (1999). Orphanet: an online rare disease and orphan drug data base.

Autoimmune polyendocrinopathy type 3. ORPHA:227982

Autoimmune polyendocrinopathy type 3 is a rare, endocrine disease characterized by autoimmune thyroid disease associated with at least one other autoimmune disease, such as type I diabetes mellitus, chronic atrophic gastritis, pernicious anemia, vitiligo, alopecia, or myasthenia gravis, but excluding Addison disease. 